\title{
Set-valued Data Anonymization Maintaining Data Utility and Data Property
}

\author{
Dedi Gunawan \\ Kanazawa University \\ Kanazawa, Ishikawa \\ dedigun@stu.kanazawa-u.ac.jp
}

\author{
Masahiro Mambo \\ Kanazawa University \\ Kanazawa, Ishikawa
}

\begin{abstract}
Set-valued database publication has been attracting much attention due to its benefit for various applications like recommendation systems and marketing analysis. However, publishing original database directly is risky since an unauthorized party may violate individual privacy by associating and analyzing relations between individuals and set of items in the published database, which is known as identity linkage attack. Generally, an attack is performed based on attacker's background knowledge obtained by a prior investigation and such adversary knowledge should be taken into account in the data anonymization. Various data anonymization schemes have been proposed to prevent the identity linkage attack. However, in existing data anonymization schemes, either data utility or data property is reduced a lot after excessive database modification and consequently data recipients become to distrust the released database.

In this paper, we propose a new data anonymization scheme, called sibling suppression, which causes minimum data utility lost and maintains data properties like database size and the number of records. The scheme uses multiple sets of adversary knowledge and items in a category of adversary knowledge are replaced by other items in the category. Several experiments with real dataset show that our method can preserve data utility with minimum lost and maintain data property as the same as original database.
\end{abstract}

\section{CCS CONCEPTS}

- Security and privacy $\rightarrow$ Data anonymization and sanitization; Database and storage security;

\section{KEYWORDS}

Set-valued database, data anonymization, sibling suppression, adversary knowledge

\section{ACM Reference Format:}

Dedi Gunawan and Masahiro Mambo. 2018. Set-valued Data Anonymization Maintaining Data Utility and Data Property. In IMCOM '18: The 12th

Permission to make digital or hard copies of all or part of this work for personal or classroom use is granted without fee provided that copies are not made or distributed for profit or commercial advantage and that copies bear this notice and the full citation on the first page. Copyrights for components of this work owned by others than the author(s) must be honored. Abstracting with credit is permitted. To copy otherwise, or republish, to post on servers or to redistribute to lists, requires prior specific permission and/or a fee. Request permissions from permissions@acm.org.

IMCOM '18, Fanuary 5-7, 2018, Langkawi, Malaysia

(C) 2018 Copyright held by the owner/author(s). Publication rights licensed to the Association for Computing Machinery.

ACM ISBN 978-1-4503-6385-3/18/01...\$15.00

https://doi.org/10.1145/3164541.3164583
International Conference on Ubiquitous Information Management and Communication, January 5-7, 2018, Langkawi, Malaysia. ACM, New York, NY, USA, 8 pages. https://doi.org/10.1145/3164541.3164583

\section{INTRODUCTION}

Publishing a set-valued database such as those containing web click log, customer transaction and trajectory data allows one to analyze its data and is beneficial for the public. An unsolved problem of publishing database to the public is that sensitive information such as personal private preference can be linked to a specific individual. Therefore, an action such as data modification to achieve data anonymity should be taken before publishing the database. Pseudonym is one simple way to protect the data. It alters certain attributes such as name or personal identification number into pseudonym to create anonymous data. This techniques have been proved insecure since an adversary can commit identity linkage attack[13] by comparing some attributes in the released database with those in other publicly available databases such as voter lists and associating a record with a specific individual. Performing data modification to achieve maximum anonymity with keeping data utility known to be NP-hard problem $[16,18]$.

From the last decade, many algorithms have been proposed to deal with problems in data anonymization. Since the problems of data anonymization are complex, each solution has its trade-off between data privacy and data utility. Generalization and suppression are frequently employed techniques to obtain data anonymity but for example employing generalization technique such as full domain generalization in database[12, 15, 19, 21] leads significant information loss although it guarantees privacy preservation. Information loss means the number of information that is reduced due to data modification.

Most of the previous studies in privacy preserving data publishing concern in relational databases. This type of databases have several attributes such as identity attribute, quasi-identifier attribute and sensitive attribute. Unlike relational database, set-valued database is a type of database consisting of tuples which have a logical form TID, UID and IID without emphasizing about the detail of how the data is stored[10], where TID means tuple id while UID and IID refer to user id and a set of items id, respectively. Thus, each person in a tuple is directly associated with a set of items. Note that tuple is also called record and we use record and tuple interchangeably.

Records or tuples in set-valued database may contain different number of items and if we consider each item as a quasiidentifier like in relational database, then it will have very high dimensionality[14]. As a result, applying $k$-anonymity will not be 
effective due to the curse of high dimensionality in set-valued database [1]. Another important aspect that is less considered in data anonymization is database properties, such as database size and item length in each tuple. Those properties refer to the number of records in database and the number of items in each record respectively.

In this paper, we propose an approach called sibling replacement to generate anonymized database which protects set-valued database from identity linkage attack with maintaining data utility and data property. The proposed approach is totally different from generalization and suppression and adopts distortion based technique where value replacement is employed. In addition, it consists of two important steps, the first is grouping the records based on adversary knowledge and the second is selecting surrogate item to replace items in adversary knowledge. The surrogate item means the selected item from the same category of the items in adversary knowledge based on hierarchical generalization tree, refer to the example in Figure 1.

The rest of paper is organized as follows. Section 2 explains a preliminaries work. Section 3 describes the method of our scheme, while experimental results are reported in section 4. Finally, section 5 concludes this work as well as gives brief direction for further research.

\section{PRELIMINARIES}

\subsection{Problem formulation}

Let $\mathcal{D}$ be a set-valued database containing $|\mathcal{D}|$ tuples. Each tuple $t \in \mathcal{D}$ contains tuple id TID, user id UID and a set IID of item id's appearing in $\mathcal{D}$. Since UID is removed before data publication, $t$ only consists of TID and IID. For any tuple $t$, IID is not an empty set. Let $I=\left\{i_{1}, i_{2}, . ., i_{k}, . ., i_{N}\right\}$ be a set of all possible item id's. IID $_{i}$ is a set of item id's of $t_{i}$.

An adversary tries to perform identity linkage attack to the database $\mathcal{D}$. We assume that an adversary has prior knowledge about some of $t \in \mathcal{D}$ (refer to the next subsection for the the detail of adversary knowledge). In order to prevent the identity linkage attack we need to eliminate adversary knowledge from $\mathcal{D}$, however, we should also keep minimum data utility lost and maintain data property.

Definition 2.1. A database $\mathcal{D}$ is said to be strong against the identity linkage attack if no adversary with partial knowledge from $I$ about up to $m$ itemsets can successfully identify the record owner of any $t \in \mathcal{D}$.

Definition 2.2. A data anonymization scheme maintaining data utility and data property is a data anonymization scheme which given a database $\mathcal{D}$, generates an anonymized database $\tilde{\mathcal{D}}$ strong against the identity linkage attack where the data utility lost in $\tilde{D}$ is kept minimum and the data property of $\tilde{D}$ is as the same as that of $\mathcal{D}$.

\subsection{Example of problem}

Consider a database $\mathcal{D}$ in Table 1 is released to a data mining company for analyzing user web access. Each URL is considered as an item. To simplify the discussion, URLs are expressed by numbers $1,2,3, . ., 12$ in Table 1.
Table 1: Example of Web Click Log Dataset $\mathcal{D}$

\begin{tabular}{lcl}
\hline Name & TID & IID \\
\hline John & $t_{1}$ & 123810 \\
Alice & $t_{2}$ & 27810 \\
Mark & $t_{3}$ & 5671012 \\
Martin & $t_{4}$ & 46791012 \\
Amar & $t_{5}$ & 135811 \\
Felix & $t_{6}$ & 2389 \\
Nita & $t_{7}$ & 1467912 \\
Marta & $t_{8}$ & 347811 \\
Tommy & $t_{9}$ & 26912 \\
Mat & $t_{10}$ & 135910 \\
\hline
\end{tabular}

Items in each record are associated with individuals and can be used to re-identify record owner if an adversary has background knowledge generated from a prior investigation. Bob working in data mining company realizes that the database contains his friends' data. Since Bob knows that recently Alice just posted her leisure photos on faceme.com $\left(i_{2}\right)$ as well as wrote her holiday activities in morningnews.com $\left(i_{8}\right)$ and Mat just posted his nice holiday's pictures in meetme.com $\left(i_{5}\right)$. Given such background knowledge, Bob can perform identity linkage attack to Alice's record $t_{2}$ with success probability around $33 \%$ since 3 out of 10 tuples, $t_{1}, t_{2}$ and $t_{6}$, contain both $i_{2}$ and $i_{8}$.

To thwart such threat, we should perform data anonymization to reduce success probability of identity linkage attack by replacing items in adversary knowledge with its surrogate item, where the surrogate item is an item belonging to the same category of the items in adversary knowledge.

\subsection{Privacy requirement Privacy protection concept}

Data owner wants to release anonymized set-valued database $\tilde{\mathcal{D}}$ from $\mathcal{D}$ to other parties, e.g. data mining company. Identity linkage attack should not be successfully performed in $\tilde{\mathcal{D}}$. The attack can be conducted if an adversary has adversary knowledge $\mathcal{A K}$ on $m$ sets of items in the database which is normally derived from some surveys or investigations prior to attacking the target. Set-valued database is said to guarantee privacy if the success probability that attacker associates records with individuals in anonymized database $\tilde{\mathcal{D}}$ is lower than that in original database $\mathcal{D}$. In addition, at the same time there is no violated record, which means there is no record that newly contains adversary knowledge by data anonymization. Let us denote the success probability of attack in original database $\mathcal{D}$ as $P(\operatorname{Succ} A t t a c k(\mathcal{D}))$ and that in anonymized database $P(\operatorname{SuccAttack}(\tilde{\mathcal{D}}))$. The number of records containing adversary knowledge is reduced by replacing items in the records with other items not contained in adversary knowledge, as a result the success probability $P(\operatorname{SuccAttack}(\tilde{\mathcal{D}}))$ of the attack after performing data anonymization will be lower than that of original database. 


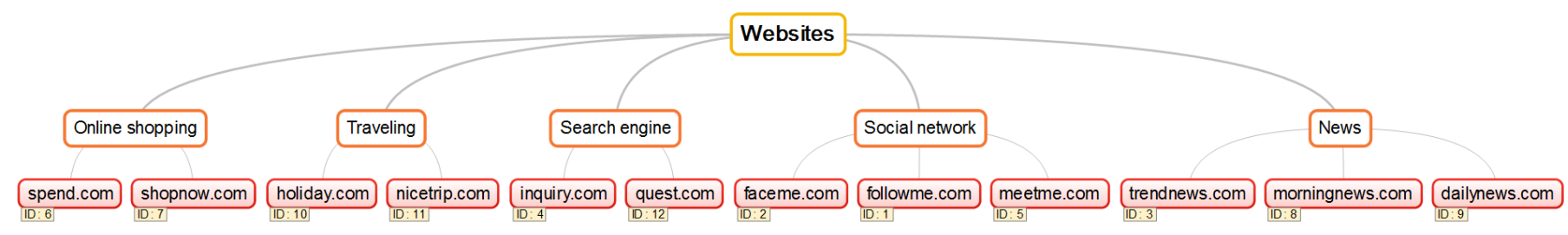

Figure 1: Hierarchical generalization.

\section{Adversary knowledge}

Considering the idea from [30], it is essential to assume that adversary may know certain sets of items in a database to attack the targeted victim. It is further assumed in [30] that adversary has unbounded background knowledge and knows both sensitive and non-sensitive items in database. There is a critique [14] that this further assumption is unrealistic since set-valued data has arbitrary items in its records. $k^{m}$-anonymity [22] assumed that adversary has knowledge about up to $m$ items from a specific record in the database. Even such an adversary cannot distinguish the record from a set of $k$ published database. In this paper we consider a candidate set $\mathcal{A} \mathcal{K}$ of adversary knowledge such that $\mathcal{A} \mathcal{K}=$ $\left\{\mathcal{A} \mathcal{K}_{1}, \mathcal{A} \mathcal{K}_{2}, \ldots, \mathcal{A} \mathcal{K}_{a}, \ldots, \mathcal{A} \mathcal{K}_{m}\right\}$ and make an assumption that an adversary obtain only one set $\mathcal{A} \mathcal{K}_{a}$ as his background knowledge. Each $\mathcal{A} \mathcal{K}_{a} \in \mathcal{A K}$ contains a set of items $i_{k}$ from $I$, where $\mathcal{A} \mathcal{K}_{a}$ $=\left\{i_{1}, i_{2}, \ldots, i_{k}, \ldots, i_{n}\right\}$. Each $\mathcal{A} \mathcal{K}_{a}$ allows the adversary to identify owner of record containing $\mathcal{A} \mathcal{K}_{a}$ with a certain success probability. Even though adversary knows a candidate set of owners corresponding to $\mathcal{A} \mathcal{K}_{a}$ we omit to express it.

\section{Information loss and dissimilarity}

The problem in data anonymization is not only guaranteeing data anonymity but also data utility after the anonymization process. There are several proposed measurement metrics to quantify data utility. In this paper we use Normalized Certainty Penalty [28] to evaluate the performance of our proposed method. We also measure the dissimilarity value between original database and anonymized database using dissimilarity metric [17].

$$
\operatorname{NCP}(i)= \begin{cases}0, & \left|u_{i}\right|=1 \\ \left|u_{i}\right| /|\mathrm{I}|, & \text { otherwise }\end{cases}
$$

where $u_{i}$ is a generalization node representing item category in hierarchical category tree and generalizes all the items under the node. The notation $\left|u_{i}\right|$ indicates the number of leaves under a generalization node $u_{i} .|I|$ refers to the number of leaves in entire hierarchical generalization tree. The $N C P$ value of item $i$ spans from 0 to $1, N C P(i)$ is 0 if there is only one leave under the generalization node $u_{i}$. Assume that the occurrences of item $i$ in database is $C_{i}$, to calculate the total $N C P$ in a database we sum up the multiplication of $N C P(i)$ to the $C_{i}$ and the sum is divided by the summation of $C_{i}$ in the database.

$$
N C P(\mathcal{D})=\frac{\sum_{i \in I} C_{i} \times N C P(i)}{\sum_{i \in I} C_{i}}
$$

Dissimilarity metric (Diss) measures the difference of data histogram between original and anonymized databases, where $f \mathcal{D}$ and $f \tilde{\mathcal{D}}$ represent the number of occurrences of item $i$ in original database and that in anonymized database respectively, while $d$ and $\tilde{d}$ are the total number of distinct items in $\mathcal{D}$ and $\tilde{D}$, respectively.

$$
\operatorname{Diss}(\mathcal{D}, \tilde{\mathcal{D}})=\frac{1}{\sum_{i=1}^{d} f \mathcal{D}(i)} \times\left|\sum_{i=1}^{d} f \mathcal{D}(i)-\sum_{i=1}^{\tilde{d}} f \tilde{D}(i)\right|
$$

\subsection{Related Work}

Several prior studies for data anonymization with certain settings and assumptions have been proposed by employing generalization and suppression. Generalization technique refers to altering original value in data attributes with more general value to obtain anonymous database. On the other hand, suppression technique tries to remove some items or records from database in order to achieve data anonymity[4].

The authors of $[25,27]$ introduced guarding node scheme based on generalization technique to protect sensitive values. For example, gastric ulcer is guarded with stomach disease and pneumonia is guarded with respiratory infection. This scheme successfully reduces the success probability that of an adversary performs identity linkage attack. Unfortunately, this type of solution reduces data size significantly for set-valued database especially when there are several items with the same category in one record. Sibling generalization scheme is proposed in [12] in order to achieve data anonymity. However, this scheme results in unnatural data since specific values are mixed with general value. Other research $[26,28]$ proposed the idea of cell generalization scheme with employing local recoding. These approaches result in less data distortion and it reduces data utility, but unfortunately flexibility leads data exploration problem[8]. Therefore, we argue that implementing generalization and suppression schemes to obtain anonymized set-valued database is more likely not suitable for maintaining data utility as well as data property since data properties such as the number of items, item length and the number of records reduce significantly. Still we stress that it is important to maintain those properties in order to avoid disbelief from data recipients.

There are several works that have contributed to set-valued data anonymization $[5,10,31]$. However, those work used either generalization or suppression, and combination of both of them to obtain anonymized database. Table 7 and 8 show the properties and features of several prior work and our proposed method in set-valued data anonymization. An anonymization scheme based on suppression technique is proposed in [29] which assumes that adversary knows a certain number of items $(p)$ for each transaction 
and removes the itemset that appears below the specified $k$ value. $\rho$-uncertainty proposed in [11] utilizes partial suppression based on heuristic approach which removes only items in sensitive association rules to avoid excessive information loss. By improving the previous work, a scheme in [5] combining local generalization and partial suppression considers the sensitivity degree of each item as the parameter value to generate anonymized database. As a trajectory data anonymization, $(K, C)_{L}$-privacy is proposed in [6] based on local suppression method which removes sequential trajectory that violates the privacy based on the given $K, C$ and $L$ values. Applying suppression technique successfully minimizes information loss. Unfortunately, it causes significant reduction with respect to database size and the length of records which may raise disbelief to the data recipients.

As an alternative of generalization and suppression schemes, we adopt the idea from blocking based technique[20] which is also successfully implemented in privacy preserving data mining area[3][24] to anonymize set-valued databases. The blocking based technique does not discard or insert additional items in a tuple; instead, it replaces sensitive items with unknown value, commonly using question mark "?" or asterisk "*”. As a result, an adversary finds it difficult to reveal the real value[23]. In this research, we do not use question mark "?" to replace items in adversary knowledge since we want to keep the anonymized database in its natural format. Thus, we use surrogate item which comes from the same category of items in adversary knowledge to generate anonymized database.

\section{DATA ANONYMIZATION PROCESS}

Before explaining proposed data anonymization process, we provide some key terms of our proposal.

\section{Victim item}

Victim item $v i_{a, k}$ is an item $i_{k} \in \mathcal{A} \mathcal{K}_{a}$ and is replaced with other item to eliminate adversary knowledge in $\mathcal{D}$. To generate an anonymized database we do not replace all the items in $\mathcal{A} \mathcal{K}_{a}$, and replace selected items $i_{k} \in \mathcal{A} \mathcal{K}_{a}$, which results in minimum dissimilarity to avoid to make excessive difference between $\mathcal{D}$ and $\tilde{\mathcal{D}}$.

\section{Surrogate item}

In existing data anonymization methods no method that replaces items with another items. In this paper we introduce an new approach that uses item replacement to generate an anonymized database. The replacement of $v i_{a, k}$ should not be done arbitrarily since it may either reduce data utility due to a lot of information lost or disrupt data property by the replacement with items already existing in the record. Therefore, selecting an appropriate item, surrogate item, for the replacement of $v i_{a, k}$ should be carefully selected with respect to data utility and data property. To this end user needs to create a hierarchical tree with depth 2 to categorize items in database. The leaves of the tree are items and the intermediate nodes are categories of items. The most general category is assigned to the root. An example of the hierarchical tree is shown in Figure 1. To derive a surrogate item $s i_{a, k}$ for replacing $v i_{a, k}$, we need to list all the siblings of $v i_{a, k}$ in the same category of the hierarchical tree to create a candidate surrogate item list, $C \mathcal{S}_{a, k}$. After creating the list we select $s i_{a, k}$ among items in $C S_{a, k}$.

\subsection{Proposed method: Sibling replacement}

The proposed method, sibling replacement, consists of two main steps to achieve a desirable anonymous database. At first, we group tuples with the same adversary knowledge $\mathcal{A} \mathcal{K}_{a}$ into one group. Second, we select a surrogate item for each item $i_{k} \in \mathcal{A} \mathcal{K}_{a}$ which has the same category with $i_{k} \in \mathcal{A} \mathcal{K}_{a}$.

\section{Record grouping}

Before performing data anonymization database $\mathcal{D}$ is split into multiple groups $G_{a}$ 's of tuples. To group the tuples, scan all the tuples $t_{i} \in \mathcal{D}$ to check whether the IID $_{i}$ contains any $\mathcal{A} \mathcal{K}_{a} \in \mathcal{A} \mathcal{K}$ $=\left\{\mathcal{A} \mathcal{K}_{1}, \mathcal{A} \mathcal{K}_{2}, . ., \mathcal{A} \mathcal{K}_{a}, . ., \mathcal{A} \mathcal{K}_{m}\right\}$. The $\mathcal{A} \mathcal{K}_{a}$ can be used to form a group $G_{a}$ of tuples. For example in Table 1 , given $\mathcal{A} \mathcal{K}_{1}=\left\{i_{2}, i_{8}\right\}$, $G_{1}=\left\{t_{1}, t_{2}, t_{6}\right\}$. In our proposed algorithm there is no intersection among these groups since the $t_{i}$ is checked based on the order of $\mathcal{A} \mathcal{K}_{a}$. For $a=1$ to $m$, check whether $\mathcal{A} \mathcal{K}_{a} \subseteq \mathbf{I I D}_{i}$ is satisfied, if it is true then add $t_{i}$ to $G_{a}$. On the other hand, the rest of the tuples not containing any $\mathcal{A} \mathcal{K}_{a}$ is grouped as $G_{r}$. Tuples of this group are not processed in $\mathcal{D}$ for data anonymization. The aims of grouping the tuples is to avoid any miss replacement of the tuples not containing any $\mathcal{A} \mathcal{K}_{a}$. Moreover, the grouping offers benefit to reduce computational time, specifically if we have multiple servers so that each group of records could be sent to each server for data anonymization process. Algorithm 1 presents a grouping method.

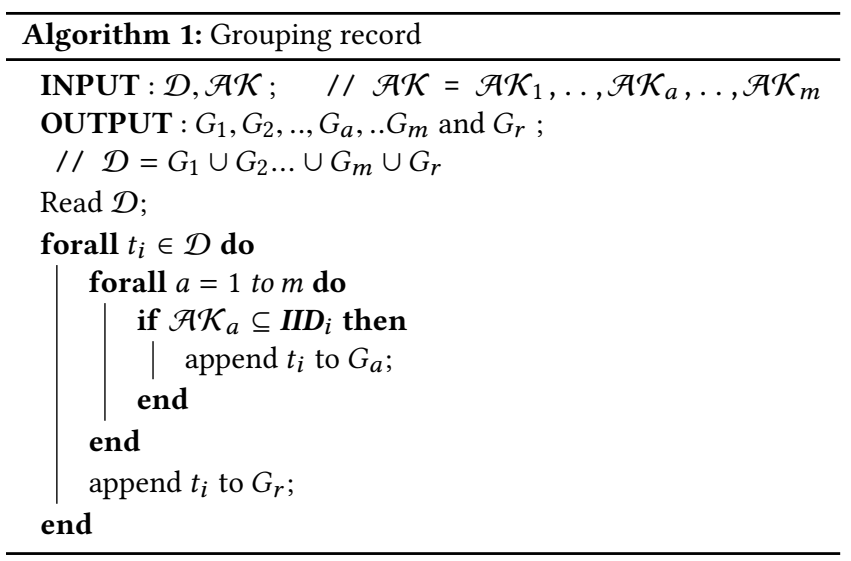

\section{Requisites of surrogate item: Violated record and Item loss}

There are some strict requisites for items in $C \mathcal{S}_{a, k}$ to be selected as $s i_{a, k}$. The aims of those requisites is to ensure the $P(\operatorname{SuccAttack}(\tilde{D}))$ is lower than $P(\operatorname{SuccAttack}(\mathcal{D}))$ and the data utility as well as data properties in the $\tilde{\mathcal{D}}$ is not too much different from that of $\mathcal{D}$. Firstly, $s i_{a, k}$ should not result in violated records. Even though some of records in $\mathcal{D}$ contain adversary knowledge $\mathcal{A} \mathcal{K}_{a}$, the replacement removes some items of $\mathcal{A} \mathcal{K}_{a}$ from the record so that identity linkage attack can no longer be performed. Still the replacement may induce other adversary knowledge $\mathcal{A} \mathcal{K}_{a}^{\prime}$ in the records and the 
attack can be launched with use of $\mathcal{A} \mathcal{K}_{a}^{\prime}$. Violated records, i.e. records newly containing such adversary knowledge after the data anonymization, should not be generated. The number of violated records caused by item $i \in C \mathcal{S}_{a, k}$ is denoted by $V R(i)$. An item $i \in C \mathcal{S}_{a, k}$ resulting in violated records will be penalized by adding one point to $V R(i)$ for each of violated records. That is, we straightforwardly count the number of records which newly contain adversary knowledge $\mathcal{A} \mathcal{K}_{a}^{\prime}$ by the replacement of $i_{k} \in \mathcal{A} \mathcal{K}_{a} \in G_{a}$ with $i \in C \mathcal{S}_{a, k}$. An item in $C \mathcal{S}_{a, k}$ with the smallest $V R(i)$ is selected as surrogate item $s i_{a, k}$ because it causes the minimum number of violated records. Secondly, $s i_{a, k}$ should minimize the number of items lost in the $\tilde{D}$ after the replacement, denoted as $I L(i)$. The value of $I L(i)$ from each candidate surrogate item $i \in C \mathcal{S}_{a, k}$ can be computed by checking the simultaneous occurrences of both $i \in G_{a}$ and $v i_{a, k}$ in tuples of $G_{a}$, and we count one for $I L(i)$ if both items exist in the same tuple. Thus, any item in $\mathcal{C} \mathcal{S}_{a, k}$ which satisfies the requisites will be selected as $s i_{a, k}$ to replace $v i_{a, k}$.

\section{Item selection and replacement}

Once the groups $G_{a}$ 's are created, the next step is performing data anonymization for each $G_{a}$ which is a partition of $\mathcal{D}$. To generate an anonymized group $\widetilde{G_{a}}$ we just need to eliminate all $i_{k} \in \mathcal{A} \mathcal{K}_{a}$ in $t_{i} \in \mathcal{D}$ satisfying $t_{i} \in G_{a}$. To this end, we load each $G_{a}$ then inspect each item $i_{k} \in \mathcal{A} \mathcal{K}_{a}$ and replace it with its surrogate item $s i_{a, k}$. The surrogate item selection process is performed by checking through all the candidate surrogate items in $\mathcal{C} \mathcal{S}_{a, k}$. There are two requirements that should be convinced by each item in $C S_{a, k}$ to be selected as the surrogate item $s i_{a, k}$. The first is that candidate surrogate item $i \in C \mathcal{S}_{a, k}$ should result in the minimum number $V R(i)$ of violated record and the second is that it should cause the least side effect with respect to the number $I L(i)$ of items lost by the replacement. A candidate surrogate item in $C \mathcal{S}_{a, k}$ with the minimum $(V R(i)+I L(i))$ is determined to be the surrogate item.

Once the group anonymization process has been completed, the next procedure is combining all the groups $\widetilde{G_{a}}$ and $G_{r}$ into one anonymized database $\tilde{\mathcal{D}}$. Algorithm 2 represents the procedure of group anonymiazation.

\subsection{Data truthfulness}

Unavoidable side effect of performing any data anonymization is the appearance of false data due to the present or absent of items in tuples. We realize that the data accuracy has an important role to support decision making. This can be discussed in other place indicating that the proposed scheme can be applied to such a situation. Prior to data modification, the probability of a user to get false data is 0 . However, after data anonymization process the probability is no longer 0 , instead it depends on the total number of modified tuples in $\tilde{\mathcal{D}}$. The probability that one gets certain number of true record $T_{t}$ and false records $T_{f}$ can be measured as follows,

$$
\operatorname{Pr}\left(T_{f}\right)=1-\left(\frac{|\tilde{\mathcal{D}}|-\left|M_{r}\right|}{|\tilde{\mathcal{D}}|}\right)
$$

where $\left|M_{r}\right|$ is the number of modified records. Then probability to get $T_{t}$ is

$$
\operatorname{Pr}\left(T_{t}\right)=1-\operatorname{Pr}\left(T_{f}\right)
$$

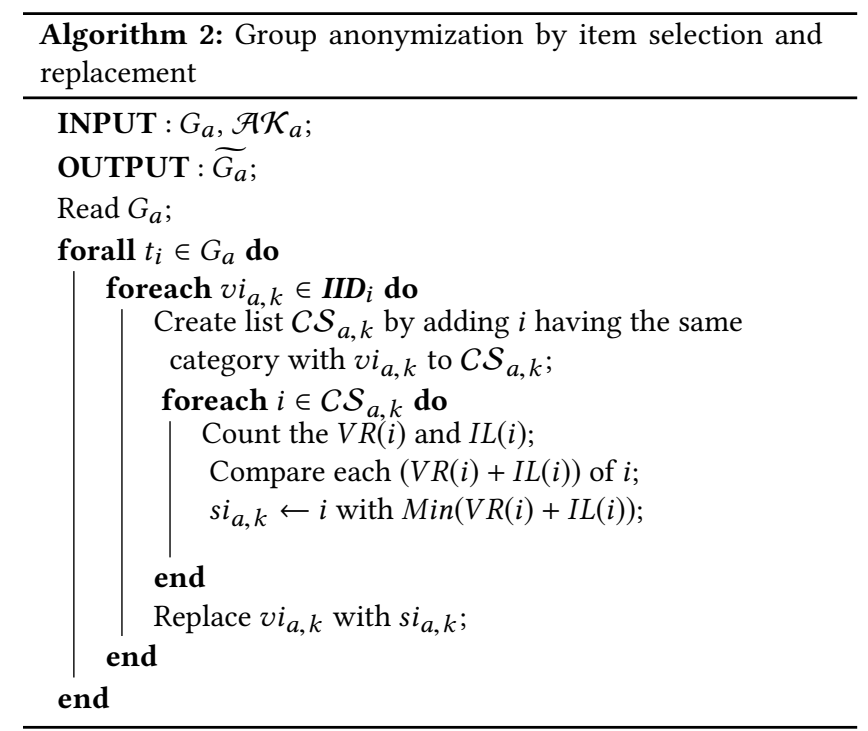

Table 2: Groups of Records $G_{1}$ (left) and $G_{2}$ (right)

\begin{tabular}{clllll}
\hline & TID & IID & & TID & IID \\
\cline { 1 - 2 } \cline { 4 - 6 }$t_{1}$ & 123810 & & 5671012 \\
$t_{2}$ & 27810 & & $t_{5}$ & 135811 \\
$t_{6}$ & 2389 \\
& & & $t_{10}$ & 135910 \\
\hline
\end{tabular}

Since the algorithm uses item substitution approach to achieve data anonymity, unavoidable side effect such as false records $t_{n} \in \tilde{D}$ may exist due to the present and absent of item $i_{k}$ from the records. Thus, we have to carefully apply the algorithm, specifically in health database or medical database where false records are not desirable.

\subsection{Example}

Refer to Section 2.2 the example of problem and associated Table 1. In the example, adversary has $m=2, \mathcal{A} \mathcal{K}_{1}=\{2,8\}$ and $\mathcal{A} \mathcal{K}_{2}=\{5\}$. Thus, the tuples in Table 1 can be grouped into 2 groups $G_{1}$ and $G_{2}$. In group $G_{1}$, there are two victim items i.e $v i_{1,2}=\{2\}$ and $v i_{1,8}=\{8\}$, while $G_{2}$ only contains one victim item $v i_{2,5}=\{5\}$. Due to space limitation, in this example we only show the anonymization process for $G_{1}$. Now, based on Figure 1 Hierarchical generalization tree we create a list of candidate surrogate items $C \mathcal{S}_{a, k}$ for each $v i_{a, k}$. The $C \mathcal{S}_{a, k}$ of $v i_{1,8}$ is item id $\{3,9\}$, while $C \mathcal{S}_{a, k}$ of $v i_{1,2}$ is item id $\{1,5\}$. Following that, we count the number of $V R(i)$ and $I L(i)$ from each candidate surrogate item $C \mathcal{S}_{a, k}$. We find that if we replace item id ' 2 ' with item id ' 1 ', it results $V R(1)=0$ and $I L(1)=1$; meanwhile, if we replace item id ' 2 ' with item id ' 5 ' it results $V R(5)=2$ and $I L(5)=0$. In this case, we select item id ' 1 ' as the surrogate item of item id '2' since it gives $\min (V R(i)+I L(i))$. After finishing group anonymization the last step is merging all $\widetilde{G_{a}}$ with $G_{r}$.

\section{EXPERIMENTAL RESULTS}

To evaluate the effectiveness of our proposed approach, we conduct several extensive experiments using real database $B M S-W e b V i e w 2$ 
Table 3: Anonymized Groups $\tilde{G}_{1}$ (left) and $\tilde{G}_{2}$ (right)

\begin{tabular}{cl}
\hline TID & IID \\
\hline$t_{1}$ & 13810 \\
$t_{2}$ & 17810 \\
$t_{6}$ & 1389 \\
\hline
\end{tabular}

\begin{tabular}{cl}
\hline TID & IID \\
\hline$t_{3}$ & 2671012 \\
$t_{5}$ & 123811 \\
$t_{10}$ & 123910 \\
\hline
\end{tabular}

Table 4: Anonymized Database $\tilde{\mathcal{D}}$

\begin{tabular}{ll}
\hline TID & IID \\
\hline$t_{1}$ & 13810 \\
$t_{2}$ & 17810 \\
$t_{3}$ & 2671012 \\
$t_{4}$ & 46791012 \\
$t_{5}$ & 123811 \\
$t_{6}$ & 1389 \\
$t_{7}$ & 1467912 \\
$t_{8}$ & 347811 \\
$t_{9}$ & 26912 \\
$t_{10}$ & 123910 \\
\hline
\end{tabular}

that contains 3,340 distinct items with 77,512 records and average length is 5 items. The data set is commonly used as the benchmark in knowledge discovery group. Following the idea of [10], we create an artificial hierarchy tree with 50 categories and each category has equally likely number of items selected from $\mathcal{D}$. All the algorithms are implemented in JAVA. To evaluate the result, we utilize $N C P$ metric to measure information loss and we use dissimilarity metric to measure the difference between original database and anonymized database. We also measure data utility for data mining tasks by counting the number of frequent itemset and rules from the anonymized database with our method and compare that of original database.

\subsection{Success probability of attack}

To confirm our proposed method, we measure the success probability of the attacker. In this experiment, we randomly generate 10 itemsets from $I$ as adversary knowledge with the length of the itemsets between 2 and 4. By using the adversary knowledge, we execute our data anonymization algorithm and found that there are 1,312 tuples contain $\mathcal{A} \mathcal{K}$. After running the algorithm, it is found that no $\mathcal{A} \mathcal{K}_{a}$ exists in $\tilde{\mathcal{D}}$. Thus, the $P(\operatorname{SuccAttack}(\tilde{\mathcal{D}}))$ is 0 . Our method can guarantee that success probability of attack in anonymized database is $0 \%$.

\subsection{Information loss and dissimilarity}

Based on the experiments, we can see in Table 5 that our proposed algorithm results in smaller information loss and dissimilarity compared with that of global generalization. It successfully reduces the information loss up to ten times lower than that of global generalization. In addition, we can achieve zero dissimilarity value, which means our method preserves data properies of the anonymized database as the same as that of original database. This is because
Table 5: Comparison of Information Loss and Dissimilarity

\begin{tabular}{llc}
\hline Method & Info.Loss & Dissimilarity \\
\hline Our method & 0.000077 & 0 \\
Global generalization & 0.00072 & 0.003 \\
\hline
\end{tabular}

Table 6: Comparison of Data Properties

\begin{tabular}{llc}
\hline Database & \# of items & Avg. length \\
\hline Original $\mathcal{D}$ & 358,278 & 4.622 \\
$\tilde{\mathcal{D}}$ with generalization & 357,237 & 4.608 \\
$\tilde{\mathcal{D}}$ with our method & 358,278 & 4.622 \\
\hline
\end{tabular}

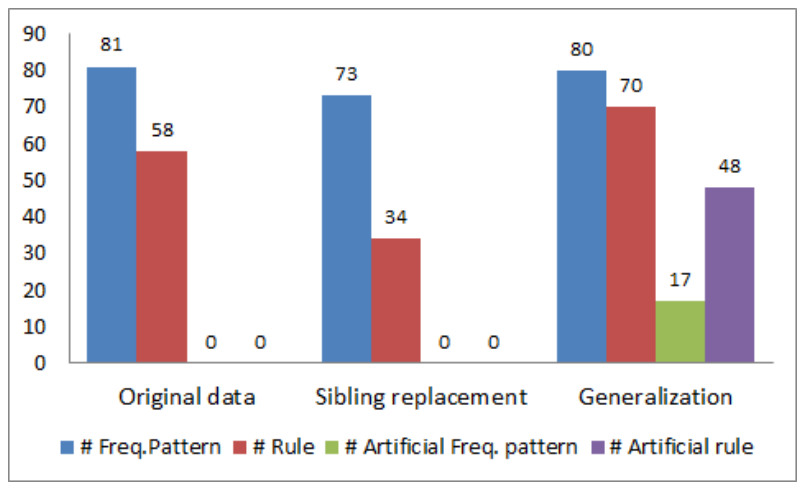

Figure 2: Data utility (supp=0.01 and $\operatorname{conf}=\mathbf{0 . 0 1})$.

items of the hierarchical tree selected only from items belonging to $\mathcal{D}$

\subsection{Data utility for data mining tasks}

To proof the data utility of our scheme, we conduct several comparisons among original database, anonymized database with our proposed sibling replacement and anonymized database with global generalization in terms of the number of frequent itemset and rules. We run data mining task such as frequent itemset mining using FP-Growth algorithm[9] and association rule mining using appriori algorithm[2] with spmf software[7] over those three databases. We also use several minimum support threshold (minSupp) ranging from 0.01 to 0.02 and minimum confident $(\min \operatorname{Con} f)$ value is 0.01 to generate frequent itemsets and rules for those mining tasks. Results from Figure 2, 3 and Figure 4 show that the number of frequent itemsets and rules from anonymized database with sibling replacement is slightly lower than that of original database. However, it does not result in artificial frequent itemset and rules. Meanwhile, anonymized database with generalization scheme results in more frequent itemsets and rules but it contains many artificial frequent itemsets and rules compared with that of original database.

\subsection{Data truthfulness}

We measure the data truthfulness for anonymized database based on the equation (4) and (5). In this experiment, we found that 1,312 
Table 7: Set-valued Data Anonymization Methods and Their Properties

\begin{tabular}{|c|c|c|c|}
\hline \multirow[b]{2}{*}{ Method } & \multicolumn{2}{|c|}{ Properties } & \multirow[b]{2}{*}{ Adversary Knowledge } \\
\hline & Anonymization technique & Utility measurement & \\
\hline$k^{m}$-anonymity & Global generalization & $\mathrm{NCP}$ & Combination of $m$-items \\
\hline$(h, k, p)$-coherence & Suppression & Frequent Itemset & Combination of itemsets \\
\hline Top-down local generalization & Local generalization & $\mathrm{NCP}$ & None \\
\hline Nonreciprocal recoding & Suppression and addition & $\begin{array}{l}\text { ILoss } \\
\text { Error rate }\end{array}$ & All non-sensitive items \\
\hline$(k, l)^{(m, n)}$-privacy & Local generalization & $\begin{array}{l}\mathrm{NCP} \\
\text { Frequent itemset }\end{array}$ & Partial non-sensitive items \\
\hline Sensitive adaptive $\rho$-uncertainty & $\begin{array}{l}\text { Local generalization and } \\
\text { suppression }\end{array}$ & $\begin{array}{l}\mathrm{NCP} \\
K L \text {-divergence } \\
\text { Frequent rule }\end{array}$ & Some items \\
\hline Our method & Substitution & $\begin{array}{l}\mathrm{NCP} \\
\text { Frequent itemset } \\
\text { Frequent rule } \\
\text { Dissimilarity }\end{array}$ & $m$-sets of itemsets \\
\hline
\end{tabular}

Table 8: Features of Set-valued Data Anonymization

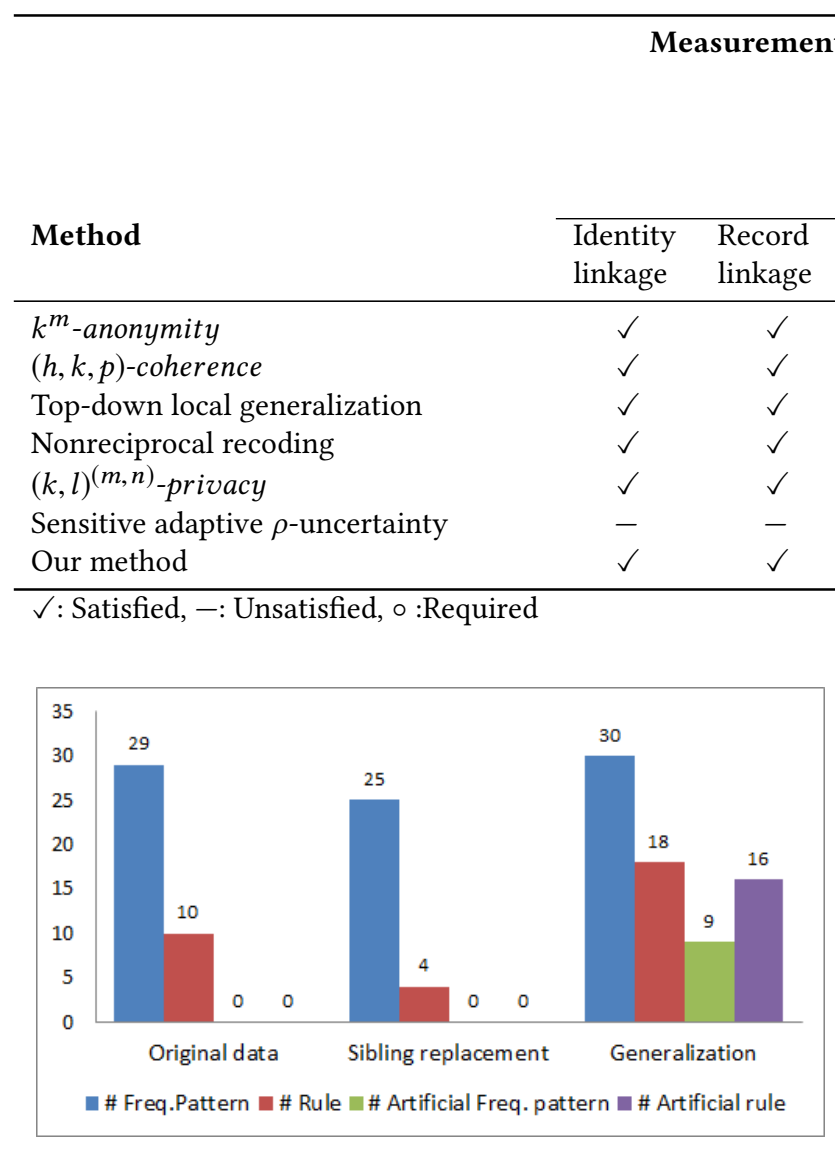

Figure 3: Data utility ( $\operatorname{supp}=\mathbf{0 . 0 1 5}$ and $\operatorname{conf}=\mathbf{0 . 0 1})$.

tuples contain $\mathcal{A K}$ and those are modified to achieve anonymized

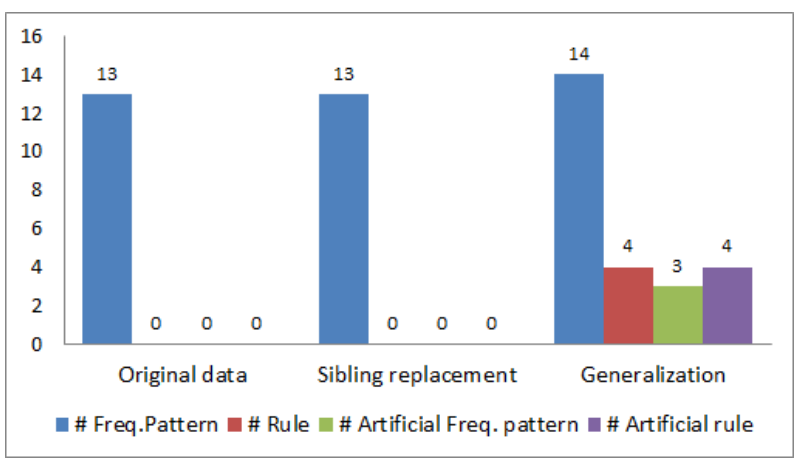

Figure 4: Data utility (supp=0.02 and $\operatorname{conf}=0.01)$.

database. Therefore, the probability of data recipient gets true data and false data are 0.984 and 0.016 , respectively. 


\section{CONCLUSION}

In this paper, we propose a data anonymization scheme maintaining data utility and data property called sibling replacement which follows blocking based approach. Unlike other existing data anonymization schemes using generalization or suppression, it uses item replacement to generate anonymized database. The proposed scheme has two main stages to generate anonymized database such as records grouping, and item selection and item replacement. Experimental results show that the proposed scheme provides lower information loss and preserves data properties as the same as that of original database. Remarkably, it does not reduce the total number of items in database (zero dissimilarity). Another advantages of this proposed algorithm is that it preserves considerable data utility for specific data mining tasks.

Item selection and replacement is the most crucial part in our anonymization scheme since it defines the quality of anonymized database. Therefore, in the future it is important to examine other selection techniques along with its criteria to have better quality of anonymized database. Another important point is modeling of adversary knowledge. Unlike possession of one adversary knowledge, adversary may perform a more elaborated attack using multiple adversary knowledge. The success probability of identity linkage attack should be also evaluated under such an adversary modeling.

\section{ACKNOWLEDGMENT}

A part of the research results has been achieved by Web-based Attack Response with Practical and Deployable Research InitiatiVE (WarpDrive), the Commissioned Research of National Institute of Information and Communications Technology (NICT), JAPAN. We would like to appreciate their support. The first author thanks to BUDI-LN scholarship from LPDP and RISTEKDIKTI.

\section{REFERENCES}

[1] Charu C Aggarwal. 2005. On K-anonymity and the Curse of Dimensionality Proceedings of the 31st International Conference on Very Large Data Bases (2005), 901-909.

[2] Rakesh Agrawal and Ramakrishnan Srikant. 1994. Fast Algorithms for Mining Association Rules in Large Databases. In Proceedings of the 20th International Conference on Very Large Data Bases (VLDB '94). Morgan Kaufmann Publishers Inc., San Francisco, CA, USA, 487-499.

[3] Maurizio Atzori, Francesco Bonchi, Fosca Giannotti, and Dino Pedreschi. 2005 Blocking Anonymity Threats Raised by Frequent Itemset Mining. In Proceedings of the Fifth IEEE International Conference on Data Mining (ICDM '05). IEEE Computer Society, Washington, DC, USA, 561-564. https://doi.org/10.1109/ICDM.2005.37

[4] Roberto J. Bayardo and Rakesh Agrawal. 2005. Data Privacy Through Optimal k-Anonymization. In Proceedings of the 21st International Conference on Data Engineering (ICDE '05). IEEE Computer Society, Washington, DC, USA, 217-228. https://doi.org/10.1109/ICDE.2005.42

[5] Liuhua Chen, Shenghai Zhong, Li-e Wang, and Xianxian Li. 2017. A SensitivityAdaptive $\rho$-Uncertainty Model for Set-Valued Data. Springer Berlin Heidelberg, Berlin, Heidelberg, 460-473. https://doi.org/10.1007/978-3-662-54970-4_27

[6] Rui Chen, Benjamin C M Fung, Noman Mohammed, Bipin C. Desai, and Ke Wang. 2013. Privacy-preserving trajectory data publishing by local suppression Information Sciences 231 (2013), 83-97. https://doi.org/10.1016/j.ins.2011.07.035

[7] Philippe Fournier-Viger, Jerry Chun-Wei Lin, Antonio Gomariz, Ted Gueniche Azadeh Soltani, Zhihong Deng, and Hoang Thanh Lam. 2016. The SPMF OpenSource Data Mining Library Version 2. Springer International Publishing, Cham, 36-40. https://doi.org/10.1007/978-3-319-46131-1_8

[8] Benjamin C. M. Fung, Ke Wang, Rui Chen, and Philip S. Yu. 2010. Privacypreserving Data Publishing: A Survey of Recent Developments. ACM Comput Surv. 42, 4, Article 14 (June 2010), 53 pages. https://doi.org/10.1145/1749603. 1749605

[9] Jiawei Han, Jian Pei, and Yiwen Yin. 2000. Mining Frequent Patterns Without Candidate Generation. SIGMOD Rec. 29, 2 (May 2000), 1-12. https://doi.org/10.
$1145 / 335191.335372$

[10] Yeye He and Jeffrey F Naughton. 2009. Anonymization of Set-Valued Data via Top-Down, Local Generalization. Proceedings of the VLDB Endowment 2, Privacy II (2009), 934-945. https://doi.org/citeulike-article-id:5897069

[11] Xiao Jia, Chao Pan, Xinhui Xu, Kenny O Zhu, and Eric Lo. 2014. $\rho$-uncertainty Anonymization by Partial Suppression. In International Conference on Database Systems for Advanced Applications. Springer, 188-202.

[12] Incognito K. LeFevre, D. DeWitt, R. Ramakrishnan. 2005. Efficient Full-Domain Kanonymity. ACM SIGMOD International Conference on Management of Data (2005).

[13] N. Li, T. Li, and S. Venkatasubramanian. 2007. t-Closeness: Privacy Beyond k-Anonymity and l-Diversity. In 2007 IEEE 23rd International Conference on Data Engineering. 106-115. https://doi.org/10.1109/ICDE.2007.367856

[14] Jun Qiang Liu. 2012. Publishing set-valued data against realistic adversaries. Journal of Computer Science and Technology 27, 1 (2012), 24-36. https://doi.org/ 10.1007/s11390-012-1203-6

[15] Ashwin Machanavajjhala, Daniel Kifer, Johannes Gehrke, and Muthuramakrishnan Venkitasubramaniam. 2007. L-diversity: Privacy Beyond K-anonymity. ACM Trans. Knowl. Discov. Data 1, 1, Article 3 (March 2007). https://doi.org/10.1145/ 1217299.1217302

[16] Adam Meyerson and Ryan Williams. 2004. On the Complexity of Optimal Kanonymity. In Proceedings of the Twenty-third ACM SIGMOD-SIGACT-SIGART Symposium on Principles of Database Systems (PODS '04). ACM, New York, NY, USA, 223-228. https://doi.org/10.1145/1055558.1055591

[17] Stanley R. M. Oliveira and Osmar R. Zaiane. 2002. Privacy Preserving Frequent Itemset Mining. In IEEE ICDM Workshop on Privacy, Security and Data Mining (PSDM 2002) (CRPIT), Chris Clifton and Vladimir Estivill-Castro (Eds.), Vol. 14. ACS, Maebashi City, Japan, 43-54.

[18] Giorgos Poulis, Grigorios Loukides, Aris Gkoulalas-Divanis, and Spiros Skiadopoulos. 2013. Anonymizing Data with Relational and Transaction Attributes. Lecture Notes in Computer Science (including subseries Lecture Notes in Artificial Intelligence and Lecture Notes in Bioinformatics) 8190 LNAI, PART 3 (2013), 353-369. https://doi.org/10.1007/978-3-642-40994-3_23

[19] P Samarati and L Sweeney. 1998. Protecting Privacy when Disclosing Information: k-Anonymity and its Enforcement Through Generalization and Suppresion. Proc of the IEEE Symposium on Research in Security and Privacy (1998), 384-393. https: //doi.org/10.1145/1150402.1150499

[20] Yücel Saygin, Vassilios S. Verykios, and Chris Clifton. 2001. Using Unknowns to Prevent Discovery of Association Rules. SIGMOD Rec. 30, 4 (Dec. 2001), 45-54. https://doi.org/10.1145/604264.604271

[21] Latanya Sweeney. 2002. K-anonymity : A Model For Protecting Privacy. International fournal of Uncertainty, Fuzziness and Knowledge-Based Systems 10, 05 (2002), 557-570. https://doi.org/10.1142/S0218488502001648 arXiv:10(5),2002;555-570

[22] M Terrovitis, N Mamoulis, and P Kalnis. 2008. Privacy-preserving anonymization of set-valued data. Proceedings of the VLDB Endowment 1, 1 (2008), 115-125. https://doi.org/10.14778/1453856.1453874

[23] V. S. Verykios, A. K. Elmagarmid, E. Bertino, Y. SaygÄśn, and E. Dasseni. 2004. Association Rule Hiding. IEEE Transactions on Knowledge and Data Engineering 16 (2004), 434-447.

[24] Vassilios S. Verykios, Emmanuel D. Pontikakis, Yannis Theodoridis, and Liwu Chang. 2007. Efficient Algorithms for Distortion and Blocking Techniques in Association Rule Hiding. Distrib. Parallel Databases 22, 1 (Aug. 2007), 85-104. https://doi.org/10.1007/s10619-007-7013-0

[25] Wei Wang, Lei Chen, and Qian Zhang. 2015. Outsourcing high-dimensional healthcare data to cloud with personalized privacy preservation. Computer Networks 88 (2015), 136-148. https://doi.org/10.1016/j.comnet.2015.06.014

[26] R C. W Wong, J Li, A W . C Fu, and K Wang. 2006. (a, k)-Anonymity: an Enhanced k-Anonymity Model for Privacy-Preserving Data Publishing. (2006).

[27] Xiaokui Xiao and Yufei Tao. 2006. Personalized privacy preservation. Vldb'06 (2006), 139-150. https://doi.org/10.1145/1142473.1142500

[28] Jian Xu, Wei Wang, Jian Pei, Xiaoyuan Wang, Baile Shi, and Ada Wai-Chee Fu. 2006. Utility-based anonymization using local recoding. ACM SIGKDD International Conference on Knowledge Discovery and Data Mining (2006), 785. https://doi.org/10.1145/1150402.1150504

[29] Yabo Xu, Benjamin C. M. Fung, Ke Wang, Ada W. C. Fu, and Jian Pei. 2008. Publishing Sensitive Transactions for Itemset Utility. In Proceedings of the 2008 Eighth IEEE International Conference on Data Mining (ICDM '08). IEEE Computer Society, Washington, DC, USA, 1109-1114. https://doi.org/10.1109/ICDM.2008.98

[30] Yabo Xu, Ke Wang, Ada Wai-Chee Fu, and Philip S. Yu. 2008. Anonymizing Transaction Databases for Publication. In Proceedings of the 14th ACM SIGKDD International Conference on Knowledge Discovery and Data Mining (KDD '08). ACM, New York, NY, USA, 767-775. https://doi.org/10.1145/1401890.1401982

[31] Mingqiang Xue, Panagiotis Karras, Chedy Raïssi, Jaideep Vaidya, and KianLee Tan. 2012. Anonymizing Set-valued Data by Nonreciprocal Recoding. In Proceedings of the 18th ACM SIGKDD International Conference on Knowledge Discovery and Data Mining (KDD '12). ACM, New York, NY, USA, 1050-1058. https://doi.org/10.1145/2339530.2339696 